\title{
Decision-making in the implementation or withdrawal of dialysis in the old complex patient
}

\author{
Nicola Panocchia, Maurizio Bossola, Luigi Tazza \\ Hemodialysis Service, Department of Surgery, Catholic University of the Sacred Heart, Rome, Italy
}

\begin{abstract}
In the last years the population of patients with end-stage renal disease has been growing and the number of patients over 74 years old on renal replacement therapy is rising. However, an increasing number of studies have shown that dialysis is not always associated with a longer life expectancy and a better quality of life for elderly patients with severe chronic comorbidity. Moreover, in selected patients conservative therapy provides a survival and quality of life comparable or even superior to that offered by dialysis. These situations pose new ethical and clinical issues. Nephrologists are increasingly faced with difficult decisions about the optimal therapeutic strategies and what is in the best interest of each patient. The new edition of the Renal Physician Association's guideline on Shared Decision-Making in the Appropriate Initiation of and Withdrawal from Dialysis takes into account these changes. For this reason the guideline advocates the use of specific parameters and tools for the prognosis assessment in order to identify the classes of patients with very poor prognosis. The importance of discussing the diagnosis, prognosis and treatment options with the patient is emphasized. Shared decision-making is the model for the physician-patient relationship. Treatment options include renal replacement therapy, not starting or stopping dialysis, and continuing medical management or palliative care. Palliative care should be offered to all patients with end-stage renal disease, whether they start or refuse dialysis and whether they continue or withdraw from dialysis. Furthermore, palliative care should be provided throughout the course of the disease, not only at the end of life.
\end{abstract}

\section{Introduction}

In contrast to the early days of dialysis, the question is now no longer, Who should be put forward for

Correspondence: Nicola Panocchia, Istituto di Clinica Chirurgica, Università Cattolica Sacro Cuore, Largo A. Gemelli 8, 00168 Roma, Italy.

Tel.: +39.06.30155485 - Fax: +39.06.30155491.

E-mail: nicola.panocchia@rm.unicatt.it

Key words: withholding and withdrawing dialysis, bioethics, elderly patients.

Conflict of interests: the authors declare that they have no potential conflict of interests.

Contributions: NP, MB, and LT made substantial contributions to the conception and design of the study, drafted the article or revised it critically for important intellectual content, and approved the final version to be published.

Received for publication: 6 April 2013.

Revision received: not required.

Accepted for publication: 7 May 2013.

This work is licensed under a Creative Commons Attribution NonCommercial 3.0 License (CC BY-NC 3.0).

CC Copyright N. Panocchia et al., 2014

Licensee PAGEPress, Italy

Italian Journal of Medicine 2014; 8:6-10

doi:10.4081/itjm.2014.172 dialysis? but, Who, if anyone, should not be put forward for dialysis? Indeed, when maintenance dialysis programs first began in the 1960s, criteria for acceptance onto dialysis were relatively strict, with this scarce resource being targeted to those most likely to benefit. Over the last 25 years, this situation has changed with growing availability of dialysis. There has been a corresponding relaxation of criteria for acceptance onto dialysis programs. Now, dialysis is offered to many who previously would not have been considered.

In developed countries, the population of patients with end-stage renal disease (ESRD) is growing steadily. The number of patients on renal replacement therapy is increasing, and it is likely that this trend will continue. The United States Renal Data System (USRDS) reported that 382,000 people were on dialysis in 2010, with the prediction that this number will increase to 533,000 by $2020 .{ }^{1}$ The INCIPE study found that the prevalence of chronic kidney disease (CKD) (all stages) was $24.7 \%$ and $44.5 \%$ in subjects over 70 and 80 years old, respectively, in a population in northeast Italy. ${ }^{2}$ The increase is not, however, uniform; a large number of affected individuals are older patients with multiple co-morbid conditions and poor functional status. Stage 3 to 5 CKD is present in $40 \%$ of people aged over 75 years, although there is some evidence that CKD may be overdiagnosed in the elderly.

The Dialysis Outcomes and Practice Patterns Study (DOPPS) on the outcome of elderly patients on 
hemodialysis showed that the proportions of dialysis patients $\geq 75$ years old were $27.4 \%$ in Europe, $20.9 \%$ in New Zealand and Australia, 16.6\% in Japan, and $23.7 \%$ in North America. In Europe, the proportion was highest in Belgium (40.7\%), followed by France (32.2\%), Sweden (28.4), Spain (27.2\%), Italy (23.9\%), and the United Kingdom (19.7\%). ${ }^{3}$ Likewise, the Lazio Regional Dialysis and Transplant Data System pointed out a dramatic change in the proportion of hemodialysis patients over the age of 74 years in the period 1994 - 2010: from $13.1 \%$ to $36.1 \%{ }^{4}$

Other important data in the literature should also be considered: i) there was no benefit from starting dialysis earlier, intended as when the glomerular filtration rate (GFR) is higher, although this approach has been most popular in recent years for older patients; 5,6 ii) dialysis treatments have significant risks, which make them dangerous, especially for certain groups of patients; ${ }^{7}$ iii) older adults with CKD are 13fold more likely to die from any cause than progress to ESRD and are 6-fold more likely to die from cardiovascular causes than develop ESRD; ${ }^{8}$ iv) the incidence of comorbidities and fragility is higher in older patients and fragility is associated with higher mortality; ${ }^{9} \mathrm{v}$ ) in nursing home residents, starting dialysis was associated with important functional decline and a very high mortality rate, ${ }^{10}$ while in patients 80 years old, the initiation of dialysis was related to the onset of disability, loss of independence and a high mortality rate; $\left.{ }^{11} \mathrm{vi}\right)$ at the end of life, patients on maintenance hemodialysis experienced higher rates of hospitalization, admission to Intensive Care Units, and use of intensive procedures than those reported for patients with cancer and heart failure; ${ }^{12}$ and vii) in the DOPPS study, withdrawal of dialysis was responsible for the death of $7.6 \%$ elderly patients in North America, $14.3 \%$ in Australia and New Zealand, 3.5\% in Europe although $0 \%$ in Japan. ${ }^{13}$ Other data showed an overall mortality rate due to dialysis suspension of nearly $25 \%$ in the United States. ${ }^{14}$

Furthermore, an increasing number of studies have shown that dialysis is not always associated with a longer life expectancy and better quality of life for elderly patients with severe chronic illness $s^{10,15}$ and that in selected patients conservative therapy provides a survival and quality of life comparable or even superior to that offered by dialysis. ${ }^{16-18}$

\section{Conservative management}

Conservative management refers to the management of a patient chooses to forego dialysis. It is named in different ways: conservative management, maximum conservative management, non-dialysis treatment, and palliative renal care. It includes active management of the renal disease in order to slow de- terioration of renal function and to minimize or relieve complications of the renal disease. It also includes active management of symptoms, fluid balance, anemia, and comorbid conditions, psychological care, social and family support, and practical help.

In a retrospective study, Chandna et al. demonstrated that in patients aged $>75$ years with many extra-renal comorbidities and a slow decline of renal function, renal replacement therapy (RRT) did not confer a significant survival advantage over that afforded by a conservative management program. ${ }^{19}$ More recently, a prospective study by the same authors showed that the median survival of patients managed conservatively was 30 months, while that of patients on RRT was 43 months. In both groups, the measures of quality of life appeared to remain stable over time, with the exception of life satisfaction, which decreased after starting dialysis. ${ }^{20}$

An observational study by Carson et al. showed that the overall median survival was longer for patients receiving RRT (37.8 months) than for those undergoing maximal conservative management (13.9 months). ${ }^{21}$ However, the rate of hospital admissions was significantly higher in RRT patients, with each RRT patient spending 25 days per year as a hospital inpatient (on average, $6.9 \%$ days of survival). Considering the days free of dialysis and hospital, the survival of the two groups was similar. Carson claimed that, for patients with an already limited life expectancy, the so-called medicalization of death is substantially greater with dialysis than without it. ${ }^{21}$

However, how long patients survive on conservative kidney management is difficult to assess since the reference point from which survival was measured varied from study to study.

\section{The new guideline}

These situations pose new ethical and clinical issues. Nephrologists are increasingly faced with difficult decisions about the optimal therapeutic strategies and what is in the best interest of each patient. The new edition of the Renal Physician Association (RPA) guideline on Shared Decision-Making in the Appropriate Initiation of and Withdrawal from Dialysis takes these changes into account. ${ }^{22}$

The guideline for adults includes ten recommendations, divided into six sections: i) the establishment of a physician-patient relationship based on the model of sharing decision-making; ii) and iii) informing patients; iv) and v) facilitating the diffusion of advance care planning; vi) and vii) making the decision whether to not initiate or to withdraw dialysis; viii) and ix) resolving conflicts about what dialysis decisions to make; and $\mathrm{x}$ ) and $\mathrm{xi}$ ) providing effective palliative care. 
Several innovations should be highlighted: the need to estimate and to discuss the prognosis with the patient; the indication to use specific parameters to assess the prognosis; the identification of classes of patients with a very poor prognosis, in whom dialysis cannot be provided safely; and the replacement of previous recommendation no. 4 on advance directives by an articulated recommendation concerning advance care planning.

For patients with CKD, a poor prognosis is determined by the presence of at least two of the following factors: i) age more than 75 years; ii) Charlson Comorbidity Index more than 8; iii) reduced autonomy determined by a Karnofsky Performance Status less than 40; and iv) severe chronic malnutrition with albumin concentrations lower than $2.5 \mathrm{~g} / \mathrm{dL}$.

Section 9 of the guideline provides numerous validated tools that clinicians may use to implement the recommendations within the guideline: a Patient Health Questionnaire to screen for depression, Trail Making Test Part B to test for cognitive impairment, the modified Charlson Comorbidity Index to calculate a comorbidity score, the Karnofsky Performance Status Scale to assess functional status, and the Dialysis Symptom Index to assess symptom frequency and severity.

Together with these risk factors, a surprise question would I be surprised if the patient died in the next year? can be used. The surprise question decreases the risk of overestimating the prognosis and has already proven to be a valuable help in identifying a poor prognosis subgroup of patients on dialysis. ${ }^{23}$

Interestingly, the new recommendation no. 6, that identifies situations in which it should be considered forgoing dialysis for acute kidney injury, CKD and ESRD, includes a new category of patients with chronic kidney failure: patients older than 75 years with two or more of the following poor prognosis criteria: i) negative response by the clinician to the surprise question (no, I would not be surprised); ii) a high comorbidity score; iii) significantly impaired functional status; iv) albumin $<2.5 \mathrm{~g} / \mathrm{dL}$. The other conditions already present in the previous edition remain unchanged: patients unable to cooperate (because of severe neurological/psychiatric disease), patients with an unstable hemodynamic status, and patients with patients with terminal illness from non-renal causes.

That determining the prognosis of elderly patients starting dialysis is felt to be clinically and ethically important is proven by the fact that a clinical scoring system to predict 6-month mortality in patients older than 75 years has been developed by French researchers. The score takes into account diabetes, low body mass, congestive heart failure, peripheral vascular disease, dysrhythmia, active malignancy, behavioral disorders, dependency on transfers, and unplanned dialysis (factors that had been independently associated with increased risk of early death). ${ }^{24}$

The guideline also indicates well-defined situations (recommendation no. 5) in which it is appropriate to forgo dialysis for patients with acute kidney injury and ESRD: fully informed patients who refuse dialysis or patients who do not have the capacity to take a decision and have previously indicated a refusal of dialysis. The recommendation includes patients with irreversible neurological impairment with loss of thought and awareness of self and environment.

\section{The decision-making process and advance care planning}

Whether the indication for starting dialysis should be considered a physician's decision or whether it should be left to the patient's desire, regardless of condition or prognosis, is a matter of great debate. ${ }^{25,26}$

Germain and Moss have argued that, although the principle of autonomy involves the right of a patient to self-determination (assuming that generally the patient is the best person to make decisions about his or her health), the doctor has the obligation to offer patient only treatments and therapies from which there is a reasonable expectation that the benefits exceed the risks. Therefore, even dialysis should be proposed only if there is a reasonable chance of reaching the objectives for the individual patient. The doctor is not forced to begin replacement kidney treatment in situations that are outside the standard of care. In addition, the existence of a negative right, such as refusing a treatment, does not imply the existence of a positive right, to be submitted to a certain treatment, if there are medical indications. ${ }^{27,28}$

The new edition of the RPA guideline emphasizes the importance of shared decision-making as a physician-patient relationship model for patients with acute and chronic renal impairment in stages IV and V. People, such as family members, who could serve as decision-makers in the case of the patient's loss of decision-making capacity should also be included in this process, with the patient's consent.

Hence, the emphasis is on the importance of information to the patient, and especially on information about the diagnosis, prognosis and treatment options. These include RRT, the option of not starting or stopping dialysis and continuing medical management or palliative care.

Shared decision-making should not mean a compromise between the perspectives of the physician and the patient. It should be a true partnership in which medical expertise meets and satisfies the patient's best interest. The relevance of evidence-based medicine (which could guide the judgment of medical futility) 
should not be ruled out but should be integrated with the patient's personal perspective.

In the new RPA guideline, advance directive is replaced by advance care planning. It has been declared that the advance directive has failed to affect patients' care..$^{29}$ Advance care planning should be offered to each patient by nephrologists and should be honored by dialysis centers except in situations in which it requires treatment contrary to standard care. Moreover, advance care planning seems to fit better in shared decision-making in the doctor-patient relationship. Indeed, advance care planning offers several advantages: determining the goals of care that are continuously discussed and verified by the patient and physician together; active and constant communication with the patient and his/her family during the progression of the disease; intervention planning based on the clinical situation and the patient's preferences; and discussion about the possibility of withdrawal from dialysis and other issues related to end-of-life care. Advance care planning seems to fit the treatments to the preferences of patients more easily, since these vary significantly over time, sometimes due to circumstantial factors, but sometimes to real changes in the core values of existence. ${ }^{30}$

\section{Palliative care}

The RPA guideline emphasizes the importance of palliative care both for patients on maintenance dialysis and for patients who choose not to start dialysis or to discontinue it. Palliative care should be offered to all patients with ESRD, whether they start or refuse dialysis, whether they continue or withdraw from dialysis. Palliative care should be provided throughout the course of the disease, not only at the end of life. The focus of palliative care is the management of symptoms and the planning of end-of-life care.

Patients on conservative management and patients who discontinue dialysis should be given the opportunity to choose to die at home or in a hospice. There is, however, little use of hospice care for patients with ERDS. Bereavement support should be offered to patients' families.

In Great Britain, the National Service for Renal Services Framework of the National Health System produced a document called End of Life Care in Advanced Kidney Disease: A Framework for Implementation, to develop a specific end-of-life care framework for patients with advanced kidney disease. The aim was to achieve high quality end-of-life care for ESRD patients in a clinical setting and promotes the empowerment of patients, helping people with advanced kidney disease to make informed choices about their needs for supportive and end-of-life care.
Achievement of this goal is dependent on promoting timely recognition of the approaching end of life; sensitive communication with patients and carers, holistic assessment and effective multi-professional work across boundaries linking kidney care, primary care, community care and palliative care services. ${ }^{31}$

\section{Ethical issues in decision-making}

Several ethical issues should be considered in decision-making. First, age per se should not be considered a criterion for withdrawing or withholding dialysis. Secondly, in patients with severe cognitive impairment, dialysis may not be carried out due to their non-cooperation. In this case withholding or suspending dialysis treatment should be motivated by respect for the dignity of the patients to avoid them unnecessary suffering. It should not be a simple denial due to their state of impaired cognition. Thirdly, social reasons (social isolation, living alone, seeming to be a burden to family members) should never be behind the decision to withhold or withdraw dialysis, otherwise there would be a risk of discrimination on a social basis. Finally, the clinician is not obliged to provide dialysis in circumstances that are outside the standard of care.

\section{Conclusions}

The debate around starting or suspending dialysis treatment has not remained confined to the medical field, but was picked up by the media. The New York Times published an article When Ailments Pile Up, Asking Patients to Rethink Free Dialysis. ${ }^{32}$ The author pointed out that the Congress of the USA established dialysis reimbursement in 1972, imagining that this would involve only 40 people per million inhabitants, aged under 55 years, without comorbidities. Currently, 400 people per million inhabitants start dialysis each year and one third of them are older than 65 years and have significant comorbidity. The cost of hemodialysis in the USA amounts to $\$ 40-50$ billion/year and $42 \%$ of this is for patients older than 65 years. The author of this article interviewed some nephrologists who stated that dialysis does not offer real benefits in terms of survival and quality of life for older people with several comorbidities.

In these years it has been observed that dialysis is not an effective treatment for all patients. In some categories of patients, dialysis does not improve either survival or quality of life and can, indeed, be burdensome. The new RPA guideline emphasizes the importance of discussing prognosis and provides tools to assess it. In this way, nephrologists should get used to not considering technical availability as a sufficient 
condition for starting dialysis. The indication for dialysis treatment should be made on the basis of careful clinical judgment, evaluated on a case-by-case basis.

However no dialysis should not be viewed as the cheap option. As Fliss Murtagh argues, The 'no dialysis' option should be regarded as a rational decision for selected patients and never as a rationing decision driven by limited resources. We need to understand, through detailed clinical research, for whom among those with ESRD the 'no dialysis' option is the most appropriate decision, and how best to deliver care that maximizes their quality of life. ${ }^{33}$

\section{References}

1. Report UAD. Atlas of CKD and ESRD. Vol 2: Atlas of ESRD. Minneapolis, MN: USRDS; 2010.

2. Gambaro G, Yabarek T, Graziani MS, et al. Prevalence of CKD in northeastern Italy: results of the INCIPE study and comparison with NHANES. Clin J Am Soc Nephrol 2010;5:1946-53.

3. Canaud B, Tong L, Tentori F, et al. Clinical practices and outcomes in elderly hemodialysis patients: results from the Dialysis Outcomes and Practice Patterns Study (DOPPS). Clin J Am Soc Nephrol 2011;6:1651-62.

4. Agenzia Sanità Pubblica Regione Lazio. Registro Dialisi e Trapianto; 2010.

5. Cooper BA, Branley P, Bulfone L, et al. A randomized, controlled trial of early versus late initiation of dialysis. N Engl J Med 2010;363:609-19.

6. Rosansky SJ. The sad truth about early initiation of dialysis in elderly patients. JAMA 2012;307:1919-20.

7. McIntyre CW, Rosansky SJ. Starting dialysis is dangerous: how do we balance the risk? Kidney Int 2012;82: 382-7.

8. Dalrymple LS, Katz R, Kestenbaum B, et al. Chronic kidney disease and the risk of end-stage renal disease versus death. J Gen Intern Med 2011;26:379-85.

9. Bao Y, Dalrymple L, Chertow GM, et al. Frailty, dialysis initiation, and mortality in end-stage renal disease. Arch Intern Med 2012;172:1071-7.

10. Kurella Tamura M, Covinsky KE, Chertow GM, et al. Functional status of elderly adults before and after initiation of dialysis. N Engl J Med 2009;361:1539-47.

11. Jassal SV, Chiu E, Hladunewich M. Loss of independence in patients starting dialysis at 80 years of age or older. N Engl J Med 2009;361:1612-3.

12. Wong SP, Kreuter W, O'Hare AM. Treatment intensity at the end of life in older adults receiving long-term dialysis. Arch Intern Med 2012;172:661-3.

13. Canaud B, Tong L, Tentori F, et al. Clinical practices and outcomes in elderly hemodialysis patients: results from the Dialysis Outcomes and Practice Patterns Study (DOPPS). Clin J Am Soc Nephrol 2011;6:1651-62.

14. USRDS annual data report. Am J Kidney Dis 2006;49:S1-S235.

15. Murtagh FE, Marsh JE, Donohoe P, et al. Dialysis or not? A comparative survival study of patients over 75 years with chronic kidney disease stage 5 . Nephrol Dial Transplant 2007;22:1955-62.
16. Brunori G, Viola BF, Parrinello G, et al. Efficacy and safety of a very-low-protein diet when postponing dialysis in the elderly: a prospective randomized multicenter controlled study. Am J Kidney Dis 2007;49:569-80.

17. De Biase V, Tobaldini O, Boaretti C, et al. Prolonged conservative treatment for frail elderly patients with end-stage renal disease: the Verona experience. Nephrol Dial Transplant 2008;23:1313-7.

18. Jassal SV, Kelman EE, Watson D. Non-dialysis care: an important component of care for elderly individuals with advanced stages of chronic kidney disease. Nephron Clin Pract 2011;119:c5-9.

19. Chandna SM, Da Silva-Gane M, Marshall C, et al. Survival of elderly patients with stage $5 \mathrm{CKD}$ : comparison of conservative management and renal replacement therapy. Nephrol Dial Transplant 2011;26:1608-14.

20. Da Silva-Gane M, Wellsted D, Greenshields H, et al. Quality of life and survival in patients with advanced kidney failure managed conservatively or by dialysis. Clin J Am Soc Nephrol 2012;7:2002-9.

21. Carson RC, Juszczak M, Davenport A, Burns A. Is maximum conservative management an equivalent treatment option to dialysis for elderly patients with significant comorbid disease? Clin J Am Soc Nephrol 2009;4:1611-9.

22. Renal Physicians Association. Shared Decision-Making in the Appropriate Initiation of and Withdrawal From Dialysis. $2^{\text {nd }}$ ed. Rockville, MD: Renal Physicians Association; 2010.

23. Moss AH, Ganjoo J, Sharma S, et al. Utility of the "surprise" question to identify dialysis patients with high mortality. Clin J Am Soc Nephrol 2008;3:1379-84.

24. Couchoud C, Labeeuw M, Moranne O, et al. A clinical score to predict 6-month prognosis in elderly patients starting dialysis for end-stage renal disease. Nephrol Dial Transplant 2009;24:1553-61.

25. Moss AH. Too many patients who are too sick to benefit start chronic dialysis nephrologists need to learn to 'just say no'. Am J Kidney Dis 2003;41:723-7.

26. Levinsky NG. Too many patients who are too sick to benefit start chronic dialysis nephrologists need to learn to 'just say no'. Con. Am J Kidney Dis 2003;41:728-32.

27. Germain MJ, Davison SN, Moss AH. When enough is enough: the nephrologist's responsibility in ordering dialysis treatments. Am J Kidney Dis 2011;58:135-43.

28. Moss AH. Ethical principles and processes guiding dialysis decision-making. Clin J Am Soc Nephrol 2011;6: 2313-7.

29. Moss AH. Revised dialysis clinical practice guideline promotes more informed decision-making. Clin J Am Soc Nephrol 2010;5:2380-3.

30. Panocchia N, Minacori R, Sacchini D, et al. Linee guida sull'inizio e la sospensione del trattamento emodialitico. Medicina \& Morale 2012;2:235-60.

31. National Health System. Kidney Care - National End of Life Program. End of life care in advanced kidney disease: a framework for implementation. Available from: http:// www.ncpc.org.uk/sites/default/files/EndOfLifeCareIn AdvancedKidneyDisease.pdf Accessed: February 15, 2013.

32. Kolata G. When ailments pile up, asking patients to rethink free dialysis. New York Times. March 31, 2011.

33. Murtagh FE, Cohen LM, Germain MJ. The "no dialysis" option. Adv Chronic Kidney Dis 2011;18:443-9. 